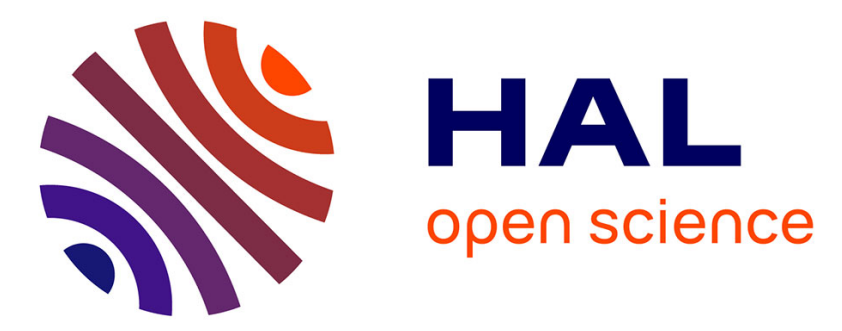

\title{
Anisotropy of Hölder Gaussian random fields: characterization, estimation, and application to image textures
}

Frédéric J P Richard

\section{- To cite this version:}

Frédéric J P Richard. Anisotropy of Hölder Gaussian random fields: characterization, estimation, and application to image textures. Statistics and Computing, 2018, 28 (6), pp.1155-1168. 10.1007/s11222017-9785-z . hal-01412095v3

\section{HAL Id: hal-01412095 \\ https://hal.science/hal-01412095v3}

Submitted on 28 Aug 2018

HAL is a multi-disciplinary open access archive for the deposit and dissemination of scientific research documents, whether they are published or not. The documents may come from teaching and research institutions in France or abroad, or from public or private research centers.
L'archive ouverte pluridisciplinaire HAL, est destinée au dépôt et à la diffusion de documents scientifiques de niveau recherche, publiés ou non, émanant des établissements d'enseignement et de recherche français ou étrangers, des laboratoires publics ou privés. 


\title{
Anisotropy of Hölder Gaussian random fields: characterization, estimation, and application to image textures.
}

\author{
Frédéric J.P. Richard
}

Richard, F.J.P. Stat Comput (2017). https://doi.org/10.1007/s11222-017-9785-z

\begin{abstract}
The characterization and estimation of the Hölder regularity of random fields has long been an important topic of Probability theory and Statistics. This notion of regularity has also been widely used in Image Analysis to measure the roughness of textures. However, such a measure is rarely sufficient to characterize textures as it does not account for their directional properties (e.g. isotropy and anisotropy). In this paper, we present an approach to further characterize directional properties associated to the Hölder regularity of random fields. Using the spectral density, we define a notion of asymptotic topothesy which quantifies directional contributions of field high-frequencies to the Hölder regularity. This notion is related to the topothesy function of the so-called anisotropic fractional Brownian fields, but is defined in a more generic framework of intrinsic random fields. We then propose a method based on multi-oriented quadratic variations to estimate this asymptotic topothesy. Eventually, we evaluate this method on synthetic data and apply it for the characterization of historical photographic papers.
\end{abstract}

Keywords Hölder regularity · anisotropy · fractional Brownian field · quadratic variations $\cdot$ texture analysis $\cdot$ photographic paper

\section{Introduction}

In this paper, we focus on irregular Gaussian random fields (called Hölder random fields) whose realizations are continuous but non-differentiable (see Section 2 for more details). The degree of Hölder regularity of these fields is quantified by a parameter $H$ (called the Hölder index) in $(0,1)$. Hölderian fields include fractional Brownian fields (i.e. multidimensional versions of fractional Brownian motions (Mandelbrot and Van Ness, 1968), and their

F. Richard

Aix Marseille Univ, CNRS, Centrale Marseille, I2M, Marseille, France.

E-mail: frederic.richard@univ-amu.fr 
anisotropic extensions (Biermé and Lacaux, 2009; Biermé et al., 2007; Bonami and Estrade, 2003; Clausel and Vedel, 2011; Richard, 2016b). They have been widely used in Image Analysis to model rough image textures from engineering domains such as Medical Imaging (Biermé et al., 2009; Biermé and Richard, 2011; Richard, 2016a; Richard and Biermé, 2010). The Hölder index of these models has served for the quantification of texture roughness.
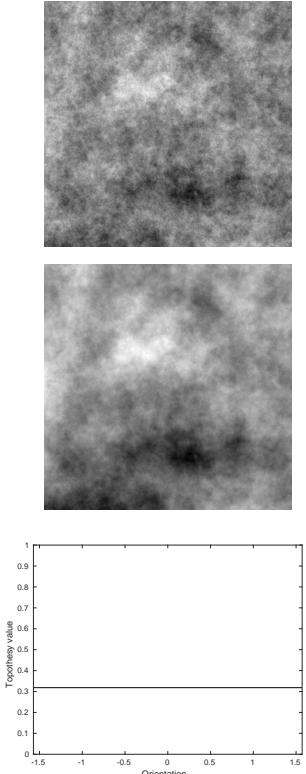
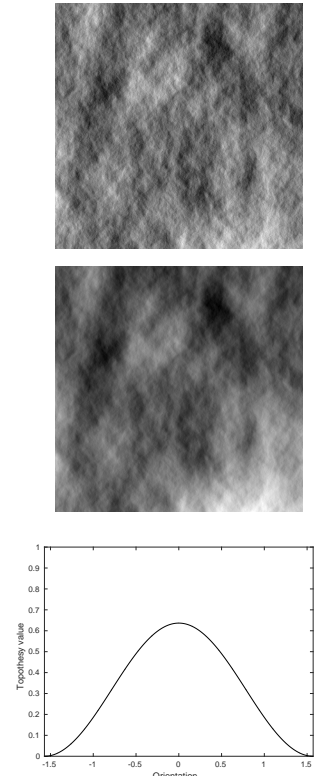
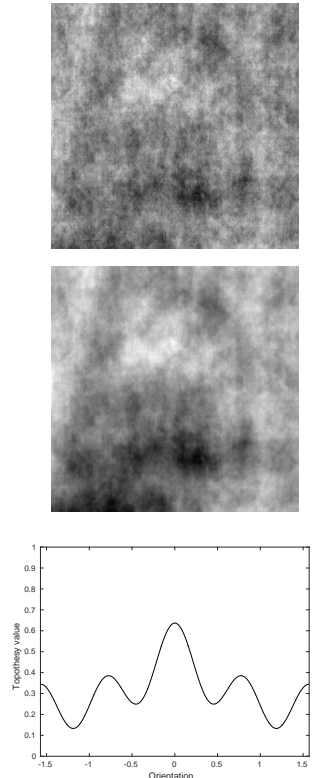

Fig. 1: Realizations of anisotropic fractional Brownian fields simulated using the turningband method of Biermé et al. (2015). Fields of first and second rows have Hölder indices of 0.3 and 0.6 , respectively. Fields of a same column have a topothesy function which is represented on the third row. Hurst functions of fields are constant and equal to the Hölder index. All simulations were obtained using a same pseudo-random number sequence so as to highlight texture dissimilarities due to variations of the simulated fields.

However, this index is not always sufficient to characterize the texture aspect. In particular, since it is regardless of orientations, it does not account for directional properties of textures. This shortcoming is illustrated on Figure 1 with some simulated textures having both low and high Hölder indices. From a regularity viewpoint, textures of the two different rows can be distinguished while those of a same row cannot. Differences between textures of a same row are only due to variations of their directional properties. In particular, the first texture of each row is isotropic (i.e. it has same aspect in all directions) whereas the second and third ones are anisotropic (i.e. their aspect varies depending on the direction). 
The main motivation for this work is to set a description of textures that would not only account for their Hölder regularity but also for relevant directional properties associated to this regularity. In that perspective, we first propose a characterization of the anisotropy of Hölder fields. Then, we address the issue of estimating features derived from this characterization.

The anisotropy of Hölder fields is often characterized through parameters of a specific model (Biermé et al., 2007; Bonami and Estrade, 2003; Clausel and Vedel, 2011; Roux et al., 2013). As an example, anisotropic fractional Brownian fields (AFBF) introduced by Bonami and Estrade (2003) are $d$ dimensional Gaussian fields with stationary increments whose second-order properties are determined by a spectral density of a form (see Section 2 for details)

$$
g_{\tau, \eta}(w)=\tau(\arg (w))|w|^{-2 \eta(\arg (w))-d} .
$$

Such a density depends on two functions $\tau$ and $\eta$ called the topothesy and Hurst functions, respectively. By assumption, these functions are both even, positive, $\pi$-periodic, and bounded functions. Depending on the spectral orientation $\arg (w)$, they can be used to characterize directional properties of AFBF; visual effects induced by the topothesy function are illustrated on Figure 1. However, such a characterization is specific to a model. Moreover, it concerns all field frequencies (including its low frequencies) and is not exclusively associated to the field regularity. More generic characterizations which are intrinsically linked to a notion of regularity were developed by Abry et al. (2015a); Hochmuth (2002); Slimane and Braiek (2012). They rely upon the analysis of an anisotropic function space (typically, an anisotropic Besov space) by decomposition of fields into an appropriate basis (e.g. a basis of the hyperbolic wavelets).

In this paper, we investigate another characterization approach which is based on the field spectral density. In a generic framework of Hölder random fields introduced by Richard (2016b), we define a function, called the asymptotic topothesy, which quantifies directional contributions of high-frequencies of a field to its irregularity. This function is related to the topothesy of an AFBF. For such a field, field high-frequencies in directions where the Hurst function reaches a minimal value $H$ are the largest. Due to these highfrequencies, the Hölder index of the field is $H$ (see Section 2 for details). The influence of these high-frequencies on the field regularity is further weighted by the topothesy function: the larger this function, the larger the high-frequencies and their contribution to the field regularity.

In a second part, we focus on the estimation of the asymptotic topothesy. This issue is both functional and non-parametric. It differs from the estimation issues that are usually tackled in the literature. Indeed, the analysis of directional features associated to random fields often reduces to the estimation of parameters of a specific anisotropic model. For instance, the estimation procedure by Roux et al. (2013), which is an implementation of the anisotropy characterization by Abry et al. (2015a) using hyperbolic wavelets, targets the single parameter of a Gaussian operator scaling field. The works of Biermé and 
Richard (2008); Richard and Biermé (2010) are devoted to the Hurst function of an AFBF. In these works, the estimation procedure is based on a Radon transform of images. Due to the discretization of this transform, the procedure can only be applied to the estimation of the Hurst function in a few directions.

Richard (2015, 2016a,b) have already investigated an estimation issue within the random field framework of this paper. In these works, an anisotropy analysis was developed using the so-called multi-oriented quadratic variations which are sums of squares of field increments computed in different directions. This analysis relies upon the estimation of a directional function which is asymptotically and linearly linked to quadratic variations. In this paper, we show that this directional function is indirectly related to the asymptotic topothesy through a convolution with a specific kernel that we analytically compute. We thus propose to recover the asymptotic topothesy of a field by solving an inverse problem associated to this convolution.

Eventually, we illustrate the interest of the asymptotic topothesy on an application to photographic papers. For the description of textures of these papers, we test a combination of two indices, an estimated Hölder index and an anisotropy index derived from the estimated asymptotic topothesy.

\section{Theoretical Framework}

\subsection{Hölder Regularity}

The definition of the asymptotic topothesy is associated to the notion of Hölder regularity which is defined as follows (Adler, 2010).

Definition 1 A field $Z$ satisfies a uniform stochastic Hölder condition of order $\alpha \in(0,1)$ if, for any compact set $C \subset \mathbb{R}^{d}$, there exists an almost surely finite, positive random variable $A$ such that the Hölder condition

$$
|Z(x)-Z(y)| \leq A|x-y|^{\alpha}
$$

holds for any $x, y \in C$, with probability one. If there exists $H \in(0,1)$ for which Condition (2) holds for any $\alpha<H$ but not for $\alpha>H$, then we say that $Z$ is Hölder of order $H$ or $H$-Hölder. The critical parameter $H$ is called the Hölder index.

Hölder fields are well-suited for the modelling of images with rough textures. In such modelling, we can interpret the texture as a visual effect of the field irregularity. Then, the texture roughness depends on the degree of the field regularity, and may be quantified from 0 to 1 by $1-H$.

For random fields with stationary increments such as the AFBF, the Hölder regularity can be characterized from the behavior of the spectral density at high-frequencies (Bonami and Estrade, 2003). 
Proposition 1 Let $Z$ is a mean square continuous Gaussian with stationary increments. Assume that its semi-variogram is determined by a spectral density $f$ as follows

$$
\frac{1}{2} \mathbb{E}\left(\left(Z(y+h)-Z(y)^{2}\right)=\frac{1}{(2 \pi)^{d}} \int_{\mathbb{R}^{d}} f(w)\left|e^{i\langle h, w\rangle}-1\right|^{2} d w .\right.
$$

Let $H \in(0,1)$.

(i) If for any $0<\alpha<H$, there exist two positive constants $A_{1}$ and $B_{1}$ such that for almost all $w \in \mathbb{R}^{d}$

$$
|w| \geq A_{1} \Rightarrow f(w) \leq B_{1}|w|^{-2 \alpha-d},
$$

then the field $Z$ is Hölder of order $\geq H$.

(ii) If, for any $H<\beta<1$, there exist two positive constants $A_{2}$ and $B_{2}$ and a positive measure subset $E$ of $[0,2 \pi)^{d-1}$ such that

$$
|w| \geq A_{2} \text { and } \arg (w) \in E \Rightarrow f(w) \geq B_{2}|w|^{-2 \beta-d},
$$

then the field $Z$ is Hölder of order $\leq H$.

(iii) If conditions (3) and (4) both hold, then the field $Z$ is H-Hölder.

Example 1 (Anisotropic fractional Brownian fields)

Let $Z$ be an AFBF as defined by Equation (1). Assume that

$$
H=\operatorname{essinf}\left\{\eta(s) \in(0,1), s \in[0,2 \pi)^{d-1}, \tau(s)>0\right\} \in(0,1),
$$

Then, the field is Hölder of order $H$. More generally, assume that the field density only satisfies

$$
|w|>A \Rightarrow 0 \leq f(w)-g_{\tau, \eta}(w) \leq C|w|^{-2 H-d-\gamma},
$$

for some positive constants $A, C$ and a spectral density $g_{\tau, \eta}$ of the form (1) (Richard, 2015, 2016a,b), then it is Hölder of an order $H$ given by (5).

According to Propositions 2.1.6 and 2.1.7 of Biermé (2005), Proposition 1 is still valid for intrinsic random fields (IRF) which extend fields with stationary increments (see Chilès and Delfiner (2012) for an introduction). In what follows, we will work in the same framework of Richard (2015, 2016a,b) composed of IRF of an arbitrary order $M$ whose density fulfills Equation (6).

\subsection{Asymptotic Topothesy}

Definition 2 (Asymptotic topothesy) Let us assume that the density of a field fulfills Condition (6) for some $H \in(0,1)$. The asymptotic topothesy is a function defined, for almost all direction $s$ of $[0,2 \pi)^{d-1}$ as

$$
\tau^{*}(s)=\lim _{\rho \rightarrow+\infty} f(\rho s) \rho^{2 H+d} .
$$


The asymptotic topothesy is a non-negative and bounded function $\tau^{*}$ which basically gives a measure of the speed at which the spectral density converges to 0 at infinity in each direction. Intuitively, such a measure quantifies the magnitude of field high-frequencies: the larger $\tau^{*}$ in a direction, the slower the density convergence and the larger high-frequencies in that direction. Since $\tau^{*}$ is bounded, the density convergence occurs at a speed which is not slower than a reference speed of order $\rho^{-2 H-d}$. According to Proposition 1 (i), this implies that the Hölder index of the field cannot be below $H$. In directions $s$ where $\tau^{*}(s)=0$ the convergence speed is faster than any speed of order $\rho^{-2 H-d}$. Hence, in these directions, the condition of Proposition 1 (ii) is not satisfied. This means that high-frequencies of these directions are not large enough to make the Hölder index of the field as low as $H$. By contrast, the convergence speed is of order $\rho^{-2 H-2}$ in directions of the set

$$
E_{0}=\left\{s, \tau^{*}(s)>0\right\} .
$$

On $E_{0}$, the condition of Proposition 1 (ii) holds, which implies that the Hölder index is exactly $H$. In other words, high-frequencies in directions of $E_{0}$ contribute to the field irregularity. Their contributions are further weighted by the asymptotic topothesy: the larger $\tau^{*}$ in a direction of $E_{0}$, the larger the contribution of high-frequencies to the field irregularity.

The asymptotic topothesy linearly depends on the variance of the field. Hence, in practice, we rather use a version of a topothesy normalized by its mean. This normalized topothesy measures relative contributions of highfrequencies to the field irregularity, and provides us with a more intrinsic information about the field anisotropy.

We will say that the field texture is isotropic when these contributions are uniform (i.e. the asymptotic topothesy is constant), and anisotropic when they are not. Let us outline that these notions of isotropy and anisotropy describe high-frequencies of the field, and are intrinsically related to its irregularity.

An IRF can always be decomposed into the sum of a smooth field and a stationary field which account for its low and high frequencies, respectively. Next, we state a proposition showing how the asymptotic topothesy characterizes the correlation structure of high-frequency part of this decomposition.

Proposition 2 Let $Z$ be an IRF whose density $f$ fulfills Condition (6) for some Hölder index $H \in(0,1)$ and asymptotic topothesy $\tau^{*}$ (see Equation (7)). Then, as $A \rightarrow+\infty$, for any $x \in \mathbb{R}^{d}$,

$$
K_{A, f}(x)-K_{A, f^{*}}(x)=o\left(A^{-2 H}\right),
$$

where $K_{A, f}$ and $K_{A, f^{*}}$ are two auto-covariances defined by

$$
K_{A, g}(x)=\frac{1}{(2 \pi)^{d}} \int_{|w|>A} e^{i\langle x, w\rangle} g(w) d w \text { and } f^{*}(w)=\tau^{*}(\arg (w))|w|^{-2 H-}(10)
$$

In this proposition, $K_{A, f}$ corresponds to the auto-covariance of the highfrequency stationary field $\tilde{Z}_{A}$ of a decomposition of $Z$. Equation (9) means 
that, when $A$ tends to $\infty$, the correlation structure of this field gets approximately the same as the one of another stationary field whose auto-covariance $K_{A}^{*}$ only depends on the Hölder index and the asymptotic topothesy.

\section{Estimation Method}

In this section, we address the issue of the estimation of the asymptotic topothesy.

\subsection{Multi-oriented Quadratic variations}

Multi-oriented quadratic variations were introduced by Richard (2016b) to construct isotropy tests, and further used by Richard (2015, 2016a) to develop anisotropy indices. The definition of these variations is based on the computation of image increments. These increments give some information about image variations at highest observed scales which are not marked by trends. Moreover, as they are computed in different orientations, they provide us with relevant directional information.

Let us assume that an image is a realization of a random field $Z$ on a grid $\llbracket 1, N \rrbracket^{d}$. Let us denote by $Z^{N}[m]=Z(m / N)$ the image intensity at position $m \in \mathbb{Z}^{d}$. Given a vector $u$ in $\mathbb{Z}^{d}$, increments in direction $\arg (u)$ at scale $|u|$ are obtained by a discrete convolution

$$
\forall m \in \mathbb{Z}^{d}, V_{u}^{N}[m]=\sum_{k \in \mathbb{Z}^{2}} v[k] Z^{N}\left[m-T_{u} k\right],
$$

with an appropriate convolution kernel $v$ and a transform $T_{u}$ which is a combination of a rotation of $\operatorname{angle} \arg (u)$ and a rescaling of factor $|u|$. The kernel is chosen so as to ensure that the convolution annihilates any polynomial of a predefined order $K$ (kernel of order $K$ ) (Richard, 2016b).

Example 2 Some two-dimensional kernels selected by Richard (2016b) for their optimality are given for $L \in \mathbb{N} \backslash\{0\}$ by

$$
v\left[l_{1}, l_{2}\right]=(-1)^{l_{1}}\left(\begin{array}{l}
L \\
l_{1}
\end{array}\right),
$$

if $\left(l_{1}, l_{2}\right) \in \llbracket 0, L \rrbracket \times\{0\}$ and 0 otherwise, $\left(\begin{array}{l}n \\ k\end{array}\right)$ standing for the binomial coefficient. Such a kernel is of order $K=L-1$.

The information provided by increments are summarized into a single random variable called quadratic variations

$$
W_{u}^{N}=\frac{1}{N_{e}} \sum_{m \in \mathcal{E}_{N}}\left(V_{u}^{N}[m]\right)^{2},
$$

where $\mathcal{E}_{N}$ is a set of cardinal $N_{e}$ containing positions $m$ where increments can be computed on grid points. To get information at different scales and 
orientations, we compute quadratic variations for different vectors $u$ indexed in a set $\mathcal{I}$ of size $n_{I}$. We gather all these variations into a single random vector $Y^{N}=\left(\log \left(W_{u_{k}}^{N}\right)\right)_{k \in \mathcal{I}}$ of $\log$-variations. The following theorem specifies the asymptotic probability distribution of $Y^{N}$.

Theorem 1 For some integer $M \geq-1$, let $Z$ be a mean-square continuous Gaussian M-IRF. Assume that its spectral density $f$ fulfills Condition (6) for some $H \in(0,1)$. Let $\tau^{*}$ asymptotic topothesy defined by Equation (7). Consider a log-variation vector $Y^{N}$ constructed using a kernel $v$ of order $K>$ $M$ and $K \geq M / 2+d / 4$ if $d>4$. For all $i \in \mathcal{I}$, define random variables $\epsilon_{i}^{N}$ such that

$$
Y_{i}^{N}=H x_{i}^{N}+\log \left(\beta_{H, \tau^{*}}\left(\arg \left(u_{i}\right)\right)\right)+\epsilon_{i}^{N},
$$

with $x_{i}^{N}=\log \left(\left|u_{i}\right|^{2} / N\right)$ and

$$
\beta_{H, \tau^{*}}(\theta)=\frac{1}{(2 \pi)^{d}} \int_{[0,2 \pi)^{d-1}} \tau^{*}(\varphi) \Gamma_{H, v}(\theta-\varphi) d \varphi=\tau^{*} \circledast \Gamma_{H, v}(\theta),
$$

where $\circledast$ stands for a circular convolution product over $[0,2 \pi)^{d-1}$, and $\Gamma_{H, v}$ is defined by

$$
\Gamma_{H, v}(\theta)=\int_{\mathbb{R}^{+}}|\hat{v}(\rho \theta)|^{2} \rho^{-2 H-1} d \rho,
$$

with $\hat{v}$ the discrete Fourier transform of $v$. Then, as $N$ tends to $+\infty$, the random vector $\left(N^{\frac{d}{2}} \epsilon_{i}^{N}\right)_{i \in \mathcal{I}}$ tends in distribution to a centred Gaussian vector.

This theorem is proved by Richard (2016b) (Theorem 3.4). The expression of $\beta_{H, \tau^{*}}$ in Equation (15) was slightly changed to highlight a convolution product.

\subsection{Inverse Problem}

In Theorem 1, the topothesy appears as a solution of Equation (15). In other words, it can be theoretically recovered by solving this equation. In practice, functions $\beta_{H, \tau^{*}}$ and $\Gamma_{H, v}$ involved in this equation are unobserved. However, they can both be estimated from log-variations. Indeed, according to Equation (14), log-variations are linearly related to the Hölder index $H$, which determines the closed form (16) of $\Gamma_{H, v}$, and an intercept function which is equal to $\log \left(\beta_{H, \tau^{*}}\right)$. Hence, following Richard (2016b), we can estimate the Hölder index and the intercept function by linear regression on log-variations. Then, we can deduce estimates of $\beta_{H, \tau^{*}}$ and $\Gamma_{H, v}$.

According to these remarks, the problem can be stated as follows. Let $\left\{\theta_{j}\right\}_{j \in \mathcal{J}}$ be an indexed set of orientations in $[0,2 \pi)^{d-1}$ formed by arguments of vectors $\left\{u_{i}\right\}_{i \in \mathcal{I}}$. Let $\tilde{\beta}_{j}$ be some unbiased estimates of $\beta_{H, \tau^{*}}\left(\theta_{j}\right)$ for $j \in \mathcal{J}$, and $\tilde{H}$ an unbiaised estimate of the Hölder index $H$. Then, for all $j \in \mathcal{J}$, Theorem 1 implies that

$$
\tilde{\beta}_{j}=\Gamma_{\tilde{H}, v} \circledast \tau\left(\theta_{j}\right)+\nu_{j}
$$


for some correlated Gaussian random variables $\nu_{j}$. Let $\Sigma$ be the covariance matrix of the random vector formed by the $\nu_{j}$.

One way to recover the topothesy $\tau$ would be to minimize a generalized least square criterion

$$
C_{0}(\tau)=\sum_{i \in \mathcal{J}} \sum_{j \in \mathcal{J}} \Sigma_{i j}^{-1}\left(\tilde{\beta}_{i}-\Gamma_{\tilde{H}, v} \circledast \tau\left(\theta_{i}\right)\right)\left(\tilde{\beta}_{j}-\Gamma_{\tilde{H}, v} \circledast \tau\left(\theta_{j}\right)\right)
$$

over a space of real $\pi$-periodic functions of $L^{2}([0,2 \pi))$. Unfortunately, this minimization problem may be ill-posed; as we shall see in practice, it may lack stability, especially when $H$ is close to 1 .

So as to fix this issue, we choose a subspace $W$ of $L^{2}\left([0,2 \pi)^{d-1}\right)$ equipped with an inner product and its associated norm $|\cdot|_{W}$. Over the space $W$, we then propose to minimize the penalized least square criterion

$$
C_{\lambda}(\tau)=C_{0}(\tau)+\lambda\left|\tau-\tau_{0}\right|_{W}^{2},
$$

where $\lambda>0$ and $\tau_{0}$ is the mean value of $\tau$ over $[0,2 \pi)^{d-1}$. The second term of this criterion penalizes variations of $\tau$ by constraining the solution to remain as close as possible to a constant. The parameter $\lambda$ sets a trade-off between this prior constraint and the fidelity of the recovered $\tau^{*}$ to observations (as measured by $C_{0}$ ). In Section 3.4, we will present a method to set this parameter optimally.

\subsection{Numerical Resolution}

In order to minimize the criterion in Equation (19), we first expand $\tau$ in an orthogonal basis $\left(\varphi_{m}\right)_{m \in \mathbb{N}}$ of $W$ with $\varphi_{0} \equiv 1$ :

$$
\forall \theta \in[0,2 \pi)^{d-1}, \tau(\theta)=\sum_{m \in \mathbb{N}} \tau_{m} \varphi_{m}(\theta),
$$

for some scalar coefficients $\tau_{m}$. From now on, we will use the same notation $\tau$ for the function and its expansion coefficients. We then approximate the solution in a finite-dimensional subspace $W^{a}$ of $W$ spanned by the first $a$ basis functions. On $W^{a}$, the penalized criterion reduces to

$$
\tilde{C}_{\lambda}^{a}(\tau)=\left(L_{\tilde{H}} \tau-\tilde{\beta}\right)^{T} \Sigma^{-1}\left(L_{\tilde{H}} \tau-\tilde{\beta}\right)+\lambda \tau^{T} R \tau,
$$

where $\tilde{\beta}$ is a column vector containing estimates of $\beta$ values, $\tau$ a vector gathering $a+1$ coefficients of decomposition in the basis of $W^{a}, L_{\tilde{H}}$ a matrix of size $|\mathcal{J}| \times a$ having terms $\varphi_{m} \circledast \Gamma_{\tilde{H}, v}\left(\theta_{j}\right)$ on the $j$ th row and $m$ th column, and $R$ a diagonal matrix having $\left(0,\left|\phi_{1}\right|_{W}, \cdots,\left|\phi_{a}\right|_{W}\right)$ on the diagonal.

The minimum of the approximated criterion $\tilde{C}_{\lambda}^{a}$ is reached at

$$
\tilde{\tau}_{\lambda}^{*}=\left(L_{\tilde{H}}^{T} \Sigma^{-1} L_{\tilde{H}}+\lambda R\right)^{-1} \Sigma^{-1} L_{\tilde{H}}^{T} \tilde{\beta}
$$


3.4 Choice of $\lambda$.

The solution $\tilde{\tau}_{\lambda}^{*}$ given by Equation $(22)$ is intended to approach the solution $\tau^{*}$ of the linear system

$$
L_{H} \tau=\beta
$$

using some estimates $\tilde{\beta}$ and $\tilde{H}$ of $\beta$ and $H$, respectively.

Next, we give some bounds for the relative bias and variance of this estimation. To get these bounds, we neglect the effect of estimating $H$, and set $L_{H} \simeq L_{\tilde{H}}$; in practice, such an approximation is compensated for by an accurate estimation of $H$ (see Section 4).

Theorem 2 Let $\tau^{*}$ be the solution of the linear system (23). Define the matrix $B=L_{H}^{T} \Sigma^{-1} L_{H}$. Let $\nu_{-}$, and $\nu_{+}, \kappa$ be the lowest and highest eigenvalues, and the 2-norm condition number of $B$, respectively. Then, the relative bias and standard deviation of $\tilde{\tau}_{\lambda}^{*}$ taken as an estimator of $\tau^{*}$ are bounded as follows.

$$
\begin{gathered}
\operatorname{BIAS}=\frac{\left|\mathbb{E}\left(\tilde{\tau}_{\lambda}^{*}\right)-\tau^{*}\right|}{\left|\tau^{*}\right|} \leq \frac{\lambda \kappa|R|_{2}}{\lambda+\nu_{+}}, \\
\operatorname{STD}=\frac{\sqrt{\operatorname{trace}\left(\mathrm{V}\left(\tilde{\tau}_{\lambda}^{*}\right)\right)}}{\left|\tau^{*}\right|} \leq \frac{\kappa \nu_{+} \sqrt{\nu_{-}} \sqrt{\operatorname{trace}\left(B^{-1}\right)}}{\sqrt{\left\langle\Sigma^{-1} \beta, \beta\right\rangle_{2}}\left(\lambda+\nu_{+}\right)}
\end{gathered}
$$

Using this theorem, we can find an optimal value of $\lambda$ minimizing the bound of the relative mean square error.

Corollary 1 In the estimation of $\tau^{*}$ by $\tilde{\tau}_{\lambda}^{*}$, the relative mean square error

$$
\operatorname{RMSE}(\lambda)=\frac{\mathbb{E}\left(\left|\tilde{\tau}_{\lambda}^{*}-\tau^{*}\right|^{2}\right)}{\left|\tau^{*}\right|^{2}}
$$

is bounded by

$$
g(\lambda)=\frac{\kappa^{2}}{\left(\lambda+\nu_{+}\right)^{2}}\left(\lambda^{2}|R|_{2}^{2}+\frac{\nu_{+}^{2} \nu_{-} \operatorname{trace}\left(B^{-1}\right)}{\left\langle\Sigma^{-1} \beta, \beta\right\rangle_{2}}\right),
$$

for all $\lambda>0$. This function reaches a global minimum at

$$
\lambda^{*}=\frac{\nu_{+} \nu_{-} \operatorname{trace}\left(B^{-1}\right)}{\left\langle\Sigma^{-1} \beta, \beta\right\rangle_{2}|R|_{2}^{2}}
$$

In practice, we set the penalization weight $\lambda$ to $\lambda^{*}$. According to Equation (26), this implies that the penalization hardens as the condition number of $B$ increases. Such an increase occurs when the Hölder index $H$ gets close to 1 (see Section 4). 


\subsection{Implementation}

In this section, we describe an implementation of the estimation procedure in two dimensions $(d=2)$. The space $W$ is defined as follows. Let $p>0$. We set $\lambda_{0}=1$ and $\varphi_{0} \equiv 1$, and for any $m \in \mathbb{N}^{*}, \nu_{2 m-1}=\nu_{2 m}=\left(1+m^{p}\right)$, $\varphi_{2 m-1}(\theta)=\cos (2 m \theta)$ and $\varphi_{2 m}(\theta)=\sin (2 m \theta)$. We define a Sobolev space

$$
W=\left\{\tau=\sum_{m \geq 0} \tau_{m} \varphi_{m}, \sum_{m \geq 0} \nu_{m} \tau_{m}^{2}<+\infty\right\},
$$

and equip it with the inner product $\langle\tau, \tilde{\tau}\rangle_{W}:=\sum_{m>0} \nu_{m} \tau_{m} \tilde{\tau}_{m}$ and its associated norm $|\tau|_{W}:=\sqrt{\langle\tau, \tau\rangle_{W}}$.

To compute the solution (22), we need to evaluate terms of $L_{H}$. For that, we use a closed-form of $\Gamma_{H}$.

Proposition 3 Let $v$ be a mono-directional increment filter, i.e. $v$ is of the form $v\left[l_{1}, l_{2}\right]=v_{1}\left[l_{1}\right]$, for $\left(l_{1}, l_{2}\right) \in \llbracket 0, L \rrbracket \times\{0\}$ and 0 otherwise. Then, for $\theta \in[0,2 \pi)$,

$$
\Gamma_{H, v}(\theta)=c \gamma_{H}(\theta)
$$

where $\gamma_{H}(\theta)=|\cos (\varphi)|^{2 H}$ and $c=2 \int_{\mathbb{R}+}\left|\hat{v}_{1}(\rho)\right|^{2} \rho^{-2 H-1} d \rho$.

To apply this formula, we compute the discrete Fourier transform $\hat{\gamma}_{\tilde{H}}$ of $\gamma_{\tilde{H}}$, and set the term $L_{\tilde{H}}$ of the $j$ th row and $m$ th column as $\hat{\gamma}_{H}\left[\left\lceil\frac{m}{2}\right\rceil\right] \varphi_{m}\left(\theta_{j}\right)$. Let us quote that we do not evaluate the constant $c$. Hence, the solution is obtained up to a constant. However, this is not a matter for our application since we only use the normalized topothesy.

To solve the inverse problem, estimates $\tilde{H}$ and $\tilde{\beta}$ of $H$ and $\beta$ are also required. In our implementation, we took ordinary least square estimates of parameters of the linear model (14) (Richard, 2015, 2016a,b). Using the $\Delta$ method and Theorem 1, it could be shown that these estimates are both unbiased and asymptotically Gaussian. We further replace $\Sigma^{-1}$ by the inverse of a covariance matrix estimate of $\tilde{\beta}$.

\section{Numerical Study}

We evaluated our estimation procedure using 10000 realizations on a grid of size $800 \times 800$ of anisotropic fractional Brownian fields (see definition in Example 1). These realizations were simulated using the turning-band method developed by Biermé et al. (2015). The Hurst function $\eta$ of each simulated field was set to a constant, which was sampled from a uniform distribution on $(0.05,0.95)$. Its topothesy was defined in the approximation space $W^{\bar{a}}$ for $\bar{a}=47$. Its expansion coefficients $\tau_{m}$ were sampled from independent centered Gaussian distributions of decreasing variances $\frac{1}{\left(1+\left\lceil\frac{m}{2}\right\rceil^{2}\right)}$. We set the coefficient $\tau_{0}=\sum_{m=1}^{a}\left|\tau_{m}\right|$ so as to ensure that the topothesy was non-negative. 


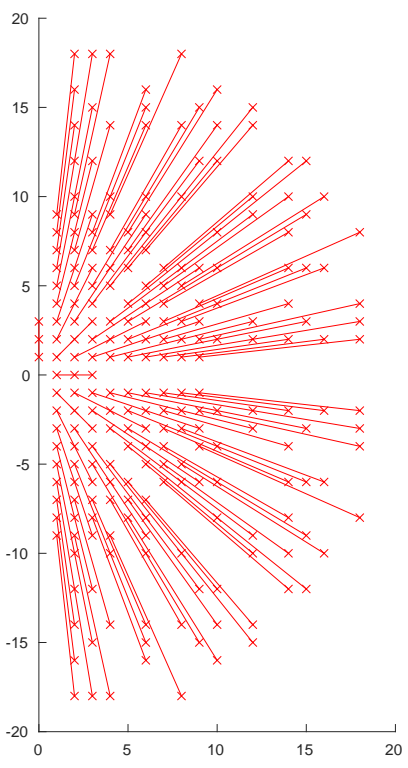

Fig. 2: Visualization of the transforms used for computing quadratic variations. Each point corresponds to a transform. The norm and argument of a point give the rescaling factor and the rotation angle of the transform, respectively. The rotation angles are further represented by segment orientations.

On each simulated field, we computed increments and quadratic variations (see Equations (11) and (13)) at scales $|u|$ and in directions $\arg (u)$ prescribed by some vectors $u$ selected in the set $\{v \in \mathbb{N} \times \mathbb{Z},|v| \in[1,20]\}$. For each represented direction, we took the three vectors with smallest scales if they existed. The set of transforms is represented on Figure 2. To compute increments, we used a kernel $v$ of the form (12) with $L=2$. Next, using these quadratic variations, we could estimate the Hölder index $H$ and the intercept function $\beta_{H, \tau^{*}}$ of Equation (15) at 96 different angles. The root mean square error for the estimation of $H$ was about $0.5 \%$. Eventually, we computed several estimates of the asymptotic topothesy by solving Equation (22) in approximation spaces $W^{a}$ of different dimensions $a \in \llbracket 1, \bar{a} \rrbracket$; we used Sobolev spaces defined by Equation (27) with different values of $p$. In Equation (22), the parameter $\lambda$ was set to the optimal value $\lambda^{*}$ given by Equation (26). For comparison, we also computed solutions obtained without penalization for $\lambda=0$. 
For each type of estimation, we evaluated the mean square error (MSE) by averaging squares of the quadratic distances between the estimated and true topothesy. We also evaluated the part of errors due to the approximation by averaging square distances between the original topothesy in the space $W^{\bar{a}}$ and its projection into an approximation space $W^{a}$ of lower dimension. These errors are plotted on Figure 3; they are expressed in percent of the mean square norm of true topothesy function.

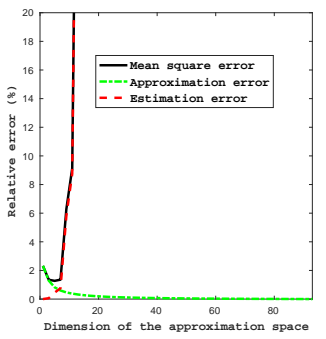

(a)

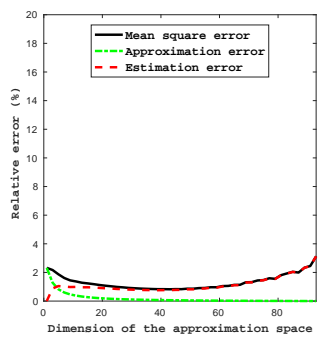

(b)

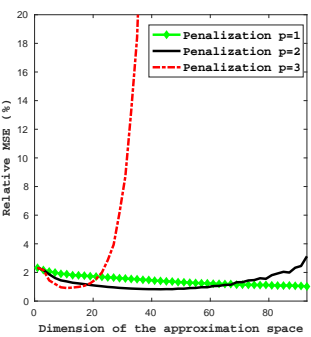

(c)

Fig. 3: Estimation errors: (a) without penalization, (b) with a penalization from Sobolev space with $p=2$, (c) with different penalizations.

We first comment Figure 3 (a) and (b). As the dimension of the approximation space was increased, the estimation error increased for both methods (with and without penalization). At lowest dimensions, the increase of these errors was compensated for by a decrease of approximation errors, leading to a decrease of MSE. Above a critical dimension, estimation errors became predominant and MSE started to increase. This critical dimension was only $a=6$ without penalization and $a=44$ with penalization. At this dimension, the minimum reached by the MSE was higher for the method without penalization $(1.27 \%)$ than for the penalization one $(0.82 \%)$. After this dimension, the MSE quickly went above $20 \%$ without penalization while it remained below $5 \%$ until the highest dimension with penalization. As a conclusion, without penalization, it was not possible to estimate correctly coefficients of high-frequency components of the topothesy. Such an estimation could be accurately achieved with a penalization, showing the benefit of the proposed method. However, as shown in Figure 3 (c), changing penalizations had an effect on the estimation. Taking a Sobolev penalization with $p=1$ slightly reduced the performance, especially at lowest dimensions, probably due to a lack of penalization. Taking a penalization with $p=3$ made the method failed at highest dimensions. For a same value of $\lambda$, the estimation bias induced by the penalization is higher for $p=3$ than for $p=2$. Hence, the optimal parameter $\lambda$ has to be set at lower values for $p=3$ than for $p=2$. As a result, the penalization can be decreased when increasing $p$. 


\begin{tabular}{|c|c|c|c|c|c|c|}
\hline$H$ & {$[0.05,0.2)$} & {$[0.2,0.35)$} & {$[0.35,0.5)$} & {$[0.5,0.65)$} & {$[0.65,0.8)$} & {$[0.8,0.95)$} \\
\hline Error (\%) & 0.59 & 0.57 & 0.62 & 0.74 & 0.93 & 1.62 \\
\hline
\end{tabular}

Table 1: Errors as a function of the Hölder index.

To further analyse results of the penalization method (with $p=2$ ), we computed MSE with an approximation dimension $a=44$ by range of Hölder index values $H$; see Table 1 . The Hölder index had an effect on the estimation performance. When it was close to 1 , the MSE was large. In this case, the condition number $\kappa$ of the matrix $L_{H}^{T} \Sigma^{-1} L_{H}$ was large, leading to a strong penalization and a high estimation bias (see Theorem 2 and Corollary 1 ). However, when $H$ was not too large $(<0.8)$ the MSE was moderate $(<1 \%)$. Hence, in such a situation, we could obtain good estimates of high frequency components of the topothesy.

\section{An application photographic papers}

In this section, we present an application to historical photographic prints. The texture of these prints is a feature which is critical for the works of artists, manufacturers, and conservators (Johnson et al., 2014; Messier et al., 2013). In particular, conservators rely upon the texture to investigate the origin of an unknown print (Johnson et al., 2014). At present, such investigations are manually done by comparing the texture of the unknow print to those of identified references. They could be eased by an automated classification tool that would select relevant references and measure texture similarities between prints. To test the feasibility of automated classifications of historic photographic paper, Johnson et al. (2014); Messier et al. (2013) assembled two datasets. The first one, named the inkjet dataset, gathers 120 photomicrographs of non-printed inkjet papers collected from the Whilhelm Analog and Digital Color Print Material Reference Collection (Messier et al., 2013). The second one, named the $\mathrm{b} \& \mathrm{w}$ dataset, is composed of 120 photomicrographs of non-printed silver gelatin photographic papers (Johnson et al., 2014). These datasets are publicly available at www.PaperTextureID.org. Some classification attempts were reported in conference papers by Abry et al. (2015b); Roux et al. (2015).

They are both organized into 4 groups containing sample sets of an increasing heterogeneity. In group 1, there are 3 sets of 10 samples obtained from a same sheet and expected by experts to have a high degree of similarity. In group 2, there are 3 sets of 10 samples from different sheets of a same manufacturer package which should also show a strong similarity albeit to a lesser extent. In group 3 , there are 3 sets of 10 samples made from same manufacturer specifications over time and expected to be more dissimilar. The group 4 is composed of 30 samples selected to show the diversity of papers. Datasets were documented by Johnson et al. (2014); Messier et al. (2013) with metadata including manufacturer, brand, date, type of texture and reflectance. 


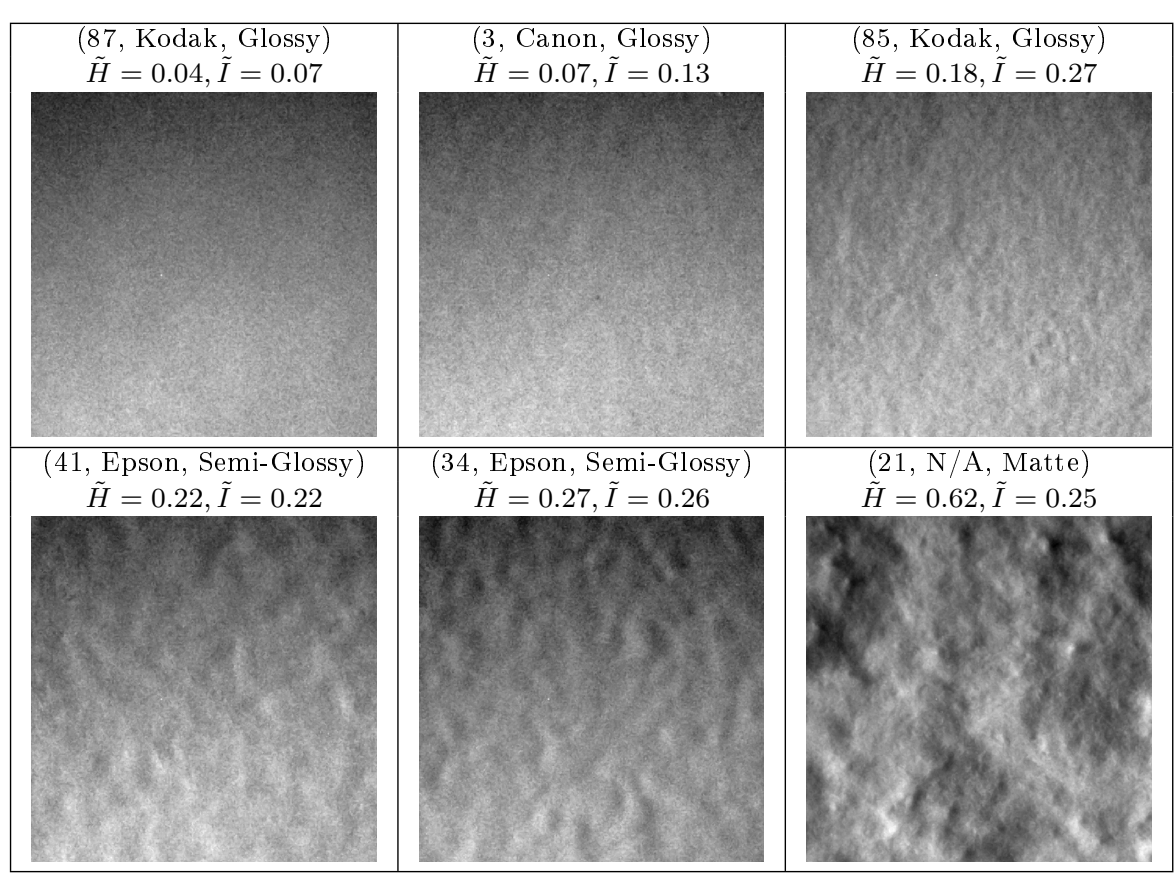

Fig. 4: Patches of size $500 \times 500$ extracted from the inkjet dataset, associated to their metadata (sample number, manufacturer, reflectance) and computed indices $\tilde{H}$ and $\tilde{I}$.

Photomicrographs of these datasets were acquired using a microscope system under the illumination of a single light placed at a 25 degree raking angle to the surface of the paper (Johnson et al., 2014; Messier et al., 2013). This specific illumination produces highlights and shadows reflecting reliefs of the paper surfaces (see examples in Figures 4 and 5). Depending on the paper properties, image textures look more or less rough and anisotropic. So as to characterize these paper properties, we used two texture features derived from our estimation procedure: the Hölder index $H$ and an anisotropy index $I$ defined as

$$
I=\sqrt{\int_{[0, \pi)}\left(\tau^{*}(s)-\int_{[0, \pi)} \tau^{*}(u) d u\right)^{2} d s}
$$

These indices were computed by replacing $H$ and $\tau^{*}$ by their estimate (see Section 3.2). Let us notice that the estimated topothesy was normalized. Consequently, the anisotropy index was invariant to both the field variance and the increment filter used for the estimation.

For the estimation of the Hölder index and the topothesy, we computed quadratic variations using a mono-directional increment filter of the form (12). The length $L$ of the filter was adapted to each image so that increments satisfy conditions of Theorem 1. It was set to $L=M+2$ (filter of order $M+1$ ) 
using an estimate $M$ of the order of the IRF underlying the image. This order $M$ was taken as the lowest one for which quadratic variations of image increments became almost constant at large scales. For most of the images, the maximal scale at which increments were computed was set to 20 pixels. But, for some papers (e.g. glossy inkjet papers), the relationship between logarithms of quadratic variations and scales was poorly linear at scales above 7 pixels. So, for these papers, the maximal scale was automatically set to 7 pixels. Between minimal and maximal scales, we used all possible increments in directions where at least two increments could be computed. Using quadratic variations of these increments, we estimated parameters of the linear model (14), including the Hölder index and the intercepts $\beta$. Using scales below 20 pixels (resp. 7 pixels), we could estimate intercepts in 96 (resp. 17) directions. Using these estimates, we set the procedure for the estimation of the topothesy function. In this procedure, the parameter $a$ was set to 47 (resp. 8) so to ensure that it was about the half of the number of intercepts. We took a Sobolev penalization with $p=2$.

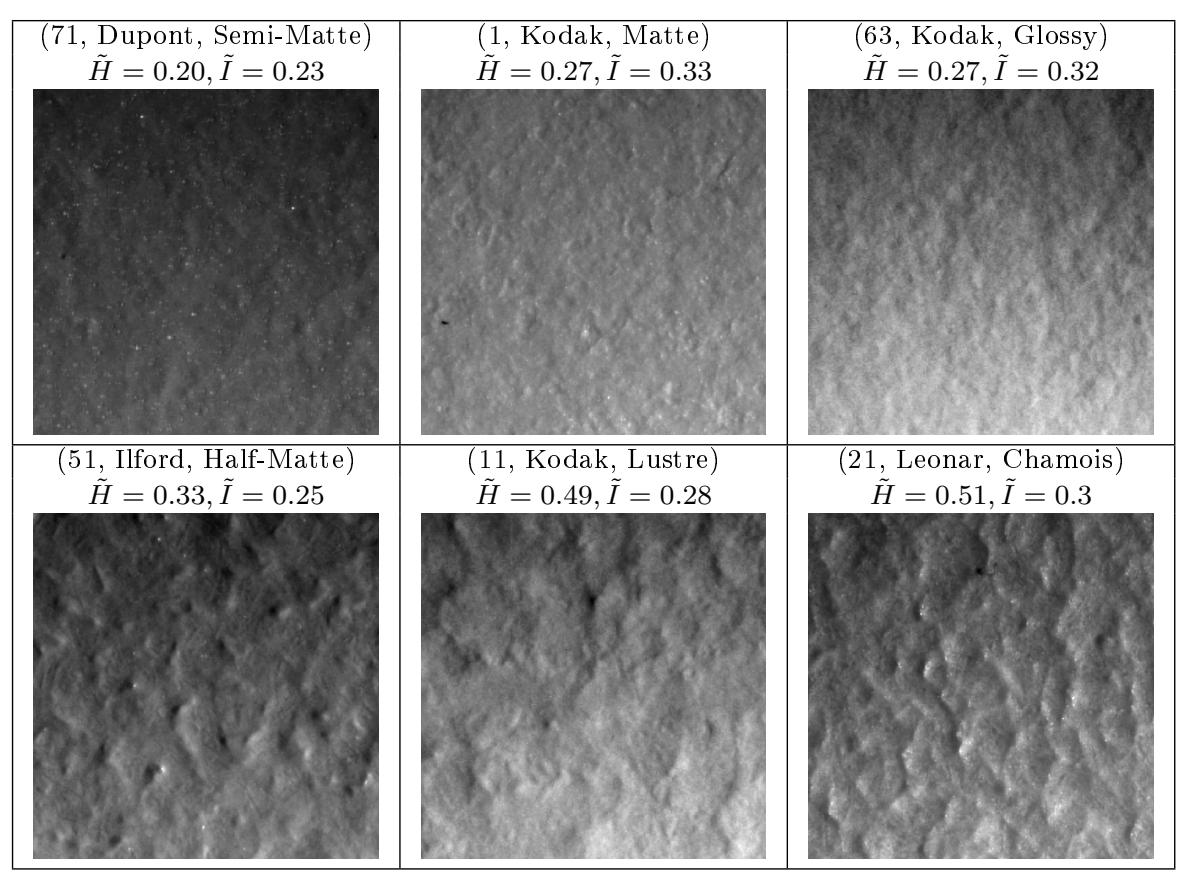

Fig. 5: Patches of size $500 \times 500$ extracted from papers of the b\&w dataset, associated to their metadata (sample number, manufacturer, reflectance) and computed indices $\tilde{H}$ and $\tilde{I}$.

On Figures 6 and 7 , we ploted couples $(\tilde{H}, \tilde{I})$ of textures of groups 1 to 3 for inkjet and b\&w datasets. For textures of group 1 ("same sheet") and 2 ("same package") of the inkjet dataset, indices were both homogeneous within 
each set and separated across sets, showing a stability of the manufacturing process. Sets $61-70$ and $71-80$ of group 3 ("same manufacturer") were also quite homogeneous, but not the set $81-90$. The variability of this set could be due to the variety of paper brands. Besides, we observed three point clusters corresponding to papers with a same reflectance: A first cluster composed of glossy papers with low Hölder and anisotropy indices, a second one containing semi-glossy papers with larger Hölder and anisotropy indices, and a third one formed by matte papers with larger Hölder indices. Some samples of these clusters are shown on Figure 4 with their associated indices. Let us note that the anisotropy index of glossy papers could be underestimated due to the use of a reduced number of topothesy coefficients in the estimation procedure.

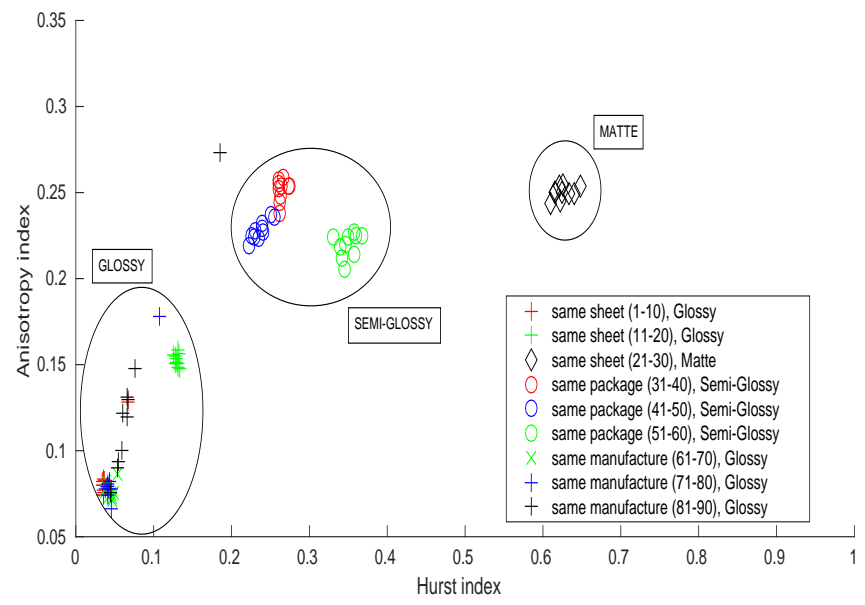

Fig. 6: Hölder and anisotropy indices of texture samples of different groups from the inkjet dataset.

Textures of the b\&w dataset showed a larger intra-set variability than those of the inkjet dataset, even for papers of groups 1 and 2. Textures of these photographic papers might be less homogeneous than the inkjet ones. The variability was particularly large on the set $81-90$ of group 3. This was probably due to the variety of paper reflectance within this set. On the b\&w 


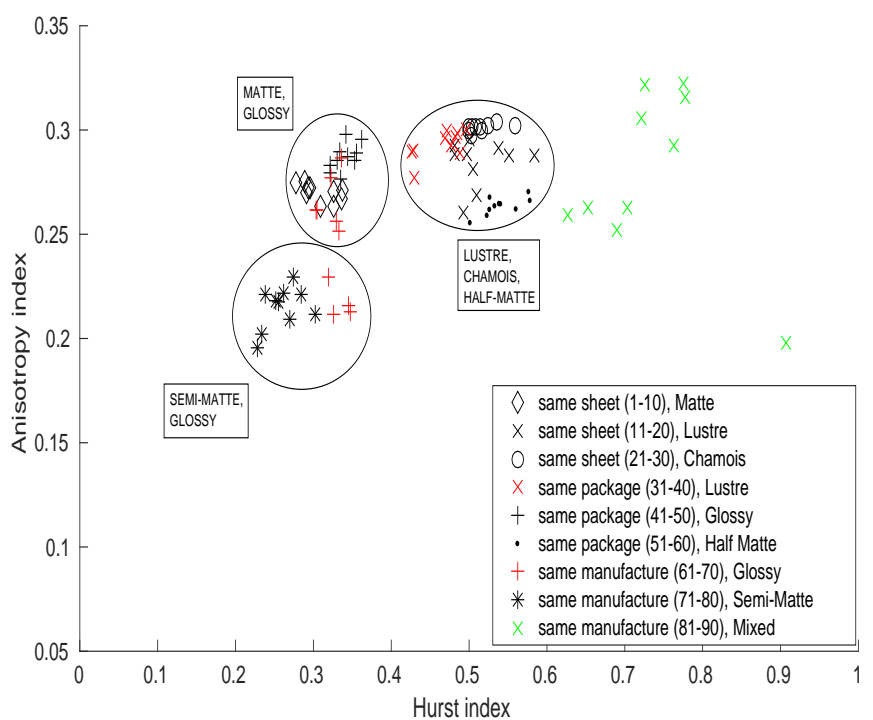

Fig. 7: Hölder and anisotropy indices of texture samples of different groups from the b\&w dataset.

dataset, we also observed three main clusters corresponding to groups of papers with common reflectance. A first cluster included the semi-matte and glossy papers (low Hölder and anisotropy indices), a second one the matte and glossy papers (low Hölder index and large anisotropy index), and a third one the lustre, champois and half-matte papers (large Hölder and anisotropy indices). Papers from these different clusters are compared on Figure 5.

For each sample pair, we further determined a degree of affinity ranging in five levels from "very poor" to "perfect". This level of affinity was computed by thresholding the euclidean distance between index values $(\tilde{H}, \tilde{I})$ of samples. In this procedure, thresholds were automatically set to optimize over a whole dataset the matching between the computed affinities and the ones established by an expert from meta-data. In Figure 8, we display in the form of a matrix the sample affinities computed for each dataset and compare them to the expert ones.

The affinity between inkjet papers of a same group was globally well estimated at level excellent, except for the group 81-90 (papers of the manufacturer Kodak). The level of affinity between glossy papers (sets 1-10 and 61-90) and 

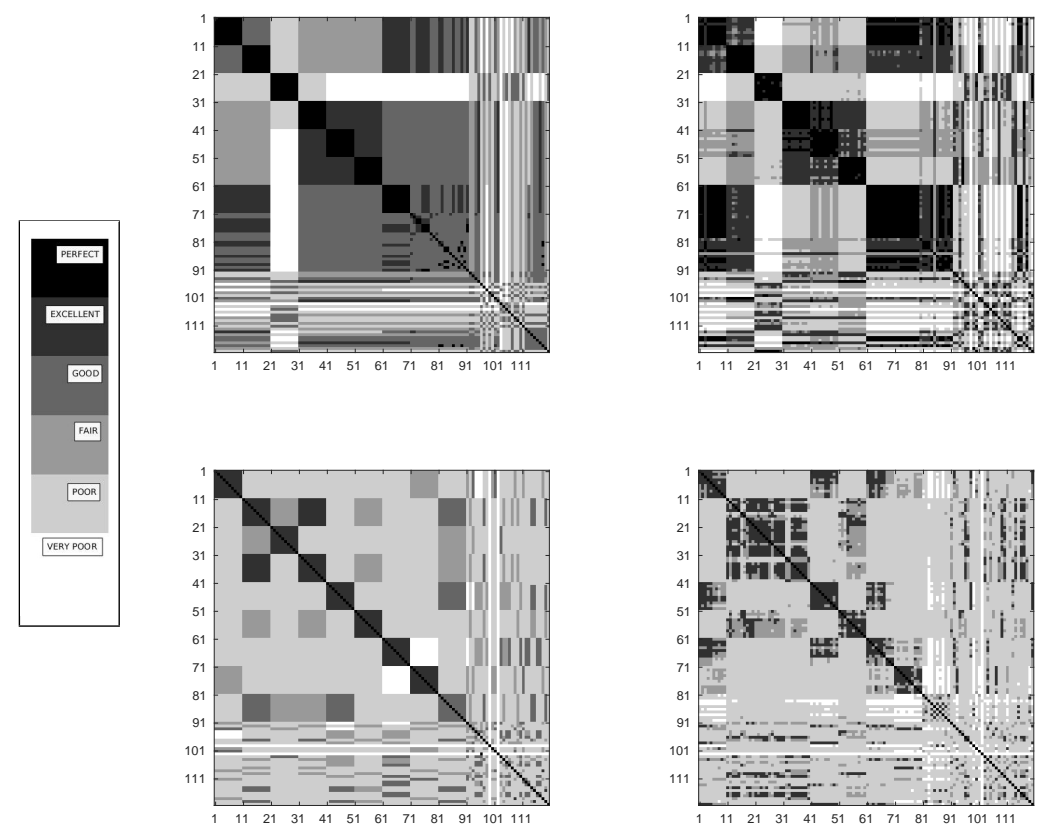

Fig. 8: Affinity matrices from experts (left) and computations (right) for the inkjet dataset (top) and b\&w dataset (bottom).

semi-glossy papers (sets 31-60), which is considered as good by experts, was underestimated at level fair or poor. The affinity between glossy papers (sets 1-10 and 61-90) and matte papers (set 21-30) was correctly estimated at level very poor whereas the one between semi-glossy papers (sets 61-90) and matte papers was slight overestimated at level poor. The affinity between glossy papers of different brands (Canon: 1-10, HP: 61:70, Epson: 71-80, Kodak: 81-90) was mostly estimated at levels excellent or perfect whereas it is only qualified as good by experts. Globally, the affinity matrices obtained by experts and the automated classification matched at a level of $40 \%$.

The affinity between b\&w papers of a same group was well-estimated at level good or excellent, except for the group 81-90 which mixes glossy and lustre papers. The affinity evaluated as poor by experts were globally wellestimated. This is for instance the case between glossy papers of Ilford group 41-50 and lustre papers from Kodak groups 11-20 and 31-40. Some affinities qualified as good by experts were underestimated at levels poor or very poor between the lustre and glossy papers of the group 81-90 and groups 11-20, 41-50. For b\&w papers, the overall match between affinity matrices obtained by experts and the automated classification was of $60 \%$. 
We also computed the affinity matrices using only the Hölder index. The degrees of match with the expert ones were $39 \%$ and $52 \%$ for inkjet and b\&w papers, respectively. The benefit of the anisotropic index was thus larger for the classification of $b \& w$ papers than inkjet papers.

\section{Discussion}

We presented an approach for the characterization of the anisotropy of Hölder random fields. Using the field spectral density, we first defined an asymptotic notion of topothesy which quantifies the directional contributions of highfrequencies to the field irregularity. We then designed and evaluated a procedure based on multi-oriented quadratic variations for the estimation of this asymptotic topothesy. Eventually, we used this procedure for the classification of textures of photographic papers. This classification was done by combining two features, a usual estimate of the Hölder index of the field and a new anisotropy index derived from the estimated asymptotic topothesy. It led to some clusters gathering papers with similar reflectances.

The anisotropy index we used are related to the ones proposed by Richard (2015, 2016a). These indices are also obtained from the directional intercept function $\beta_{H, \tau^{*}}$ that appears in the linear asymptotic relationship between multi-oriented quadratic variations and the Hölder index of the field (see Equation (15) of Theorem 1). They measure a dispersion of the intercept function and, indirectly, of the asymptotic topothesy. However, they depend on the Hölder index of the field and the order of increments required for its analysis (this order has to be set with respect to the order of the field). Hence, their variations do not exclusively reflect differences between field directional properties. They also account for changes of field regularity or order. By contrast, the anisotropy index of this paper is a direct measure of dispersion of the asymptotic topothesy. This measure is intrinsically related to directional properties of the field and invariant to its order and Hölder index.

For the classification, information carried by the asymptotic topothesy was reduced to a single anisotropy index, but it is much richer. Depending on the interest for the application, other indices such as kurtosis or skewness of the asymptotic topothesy could be computed. Directions where the asymptotic topothesy reaches optima could also be of interest for finding main orientations of an image texture. Eventually, some dimensionality reduction algorithms could be directely applied to topothesy coefficients to extract the most relevant information to be used for classification.

Besides, according to Proposition 2, the asymptotic topothesy provides us with an information about the correlation structure of field high-frequencies. Such an information could be used to set an IRF model where the image would be decomposed into a trend field and a texture field with a specified correlation structure. Using such a model, it would become possible to achieve other image processing tasks such as separation of trend and texture of an image, examplar- 
based texture simulations, or inpainting of missing or occluded parts of an image.

Our characterization and estimation approach is only devoted to the global analysis of homogeneous random fields whose Hurst and topothesy functions remain spatially constant. It would be challenging to extend it to the local analysis of heterogeneous anisotropic fields. Such an extension is analogous to the one that passed from a global regularity analysis of fractional Brownian motions to a local one of multifractional Brownian motions (see for instance Istas and Lang (1997)) To achieve it, it would be necessary to set a suitable framework of heterogeneous anisotropic fields, which can not be intrinsic anymore. It would also imply to reduce the analysis area to small neighborhoods of points, which can be critical for the estimation.

The anisotropy characterization we proposed is valid for any field of the framework, no matter how it is observed. However, our estimation procedure is specifically designed for fields observed on a lattice. Due to this particular structure, observations can be analyzed without any interpolation using lattice-preserving transforms. An extension of this procedure to partial observations could be envisaged. However, it would probably require an interpolation of data, which could have an effect on the estimation.

\section{References}

Abry, P., Clausel, M., Jaffard, S., Roux, S., Vedel, B.: Hyperbolic wavelet transform: an efficient tool for multifractal analysis of anisotropic textures. Rev. Mat. Iberoam. 31(1), 313-348 (2015a)

Abry, P., Roux, S., Wendt, H., Messier, P., et al.: Multiscale anisotropic texture analysis and classification of photographic prints. IEEE Signal Process. Mag. 32(4), 18-27 (2015b)

Adler, R.: The geometry of random fields, vol. 62. SIAM (2010)

Biermé, H.: Champs aléatoires : autosimilarité, anisotropie et étude directionnelle. Ph.D. thesis, University of Orleans, France (2005)

Biermé, H., Benhamou, C., Richard, F.: Parametric estimation for Gaussian operator scaling random fields and anisotropy analysis of bone radiograph textures. In: Pohl, K. (ed.) Proc. of MICCAI, pp. 13-24. London, UK (2009)

Biermé, H., Lacaux, C.: Hölder regularity for operator scaling stable random fields. Stoch. Proc. Appl. 119(7), 2222-2248 (2009)

Biermé, H., Meerschaert, M.M., Scheffler, H.P.: Operator scaling stable random fields. Stoch. Proc. Appl. 117(3), 312-332 (2007)

Biermé, H., Moisan, M., Richard, F.: A turning-band method for the simulation of anisotropic fractional Brownian field. J. Comput. Graph. Statist. 24(3), 885-904 (2015)

Biermé, H., Richard, F.: Estimation of anisotropic Gaussian fields through Radon transform. ESAIM: Probab. Stat. 12(1), 30-50 (2008) 
Biermé, H., Richard, F.: Analysis of texture anisotropy based on some Gaussian fields with spectral density. In: Bergounioux, M. (ed.) Mathematical Image Processing, pp. 59-73. Springer Proc. (2011)

Bonami, A., Estrade, A.: Anisotropic analysis of some Gaussian models. J. Fourier Anal. Appl. 9, 215-236 (2003)

Chilès, J., Delfiner, P.: Geostatistics: modeling spatial uncertainty. J. Wiley, 2nd ed. (2012)

Clausel, M., Vedel, B.: Explicit construction of operator scaling Gaussian random fields. Fractals 19(01), 101-111 (2011)

Hochmuth, R.: Wavelet characterizations for anisotropic Besov spaces. Appl. Comput. Harmon. Anal. 12(2), 179-208 (2002)

Istas, J., Lang, G.: Quadratic variations and estimation of the local Holder index of a Gaussian process. Ann. Inst. Henri Poincaré, Prob. Stat. 33(4), 407-436 (1997)

Johnson, R., Messier, P., Sethares, W., et al.: Pursuing automated classification of historic photographic papers from raking light images. J. Am. Inst. Conserv. 53(3), 159-170 (2014)

Mandelbrot, B.B., Van Ness, J.: Fractional Brownian motion, fractionnal noises and applications. SIAM Rev. 10, 422-437 (1968)

Messier, P., Johnson, R., Wilhelm, H., Sethares, W., Klein, A., et al.: Automated surface texture classification of inkjet and photographic media. In: NIP \& Digital Fabrication Conference, pp. 85-91. Society for Imaging Science and Technology (2013)

Richard, F.: Analysis of anisotropic Brownian textures and application to lesion detection in mammograms. Procedia Environ. Sci. 27, 16-20 (2015)

Richard, F.: Some anisotropy indices for the characterization of Brownian textures and their application to breast images. Spat. Stat. 18, 147-162 (2016a)

Richard, F.: Tests of isotropy for rough textures of trended images. Stat. Sinica 26(3), 1279-1304 (2016b)

Richard, F., Biermé, H.: Statistical tests of anisotropy for fractional Brownian textures. Application to full-field digital mammography. J. Math. Imaging Vis. 36(3), 227-240 (2010)

Roux, S., Clausel, M., Vedel, B., Jaffard, S., Abry, P.: Self-similar anisotropic texture analysis: The hyperbolic wavelet transform contribution. IEEE Trans. Image Process. 22(11), 4353-4363 (2013)

Roux, S., Tremblay, N., Borgnat, P., Abry, P., Wendt, H., Messier, P.: Multiscale anisotropic texture unsupervised clustering for photographic paper. In: IEEE Int. Workshop on Information Forensics and Security (WIFS), pp. 1-6 (2015)

Slimane, M.B., Braiek, H.B.: Directional and anisotropic regularity and irregularity criteria in Triebel wavelet bases. J. Fourier Anal. Appl. 18(5), 893-914 (2012) 


\section{A Proofs}

Proof (Proposition 2) For some positive constant $c$, we have

$$
\Delta_{A}(x)=\left|K_{A, f}(x)-K_{A, f *}(x)\right| \leq c \int_{|w| \geq A}\left|f(w)-f^{*}(w)\right| d w .
$$

Since the field density satisfies Condition (6), we further obtain

$$
\begin{aligned}
\Delta_{A}(x) & \leq c \int_{|w| \geq A}\left|f(w)-g_{\tau, \eta}(w)\right| d w+\int_{|w| \geq A}\left|f^{*}(w)-g_{\tau, \eta}(w)\right| d w \\
& \leq \tilde{c} \int_{|w| \geq A}|w|^{-2 H-d-\gamma} d w+\int_{|w| \geq A}\left|f^{*}(w)-g_{\tau, \eta}(w)\right| d w \\
& \leq o\left(A^{-2 H}\right)+\int_{|w| \geq A}\left|f^{*}(w)-g_{\tau, \eta}(w)\right| d w
\end{aligned}
$$

as $A$ tends to $+\infty$.

Now, in directions $s$ of the set $E_{0}=\left\{s, \tau^{*}(s)>0\right\}$, we notice that $\eta(s)=H$ and $\tau^{*}(s)=\tau(s)$. Hence, $f^{*}(w)=g_{\tau, \eta}(w)$ whenever $\arg (w)$ is in $E_{0}, f^{*}(w)=g_{\tau, \eta}(w)$, and 0 otherwise. Consequently, in polar coordinate, we have

$$
\begin{aligned}
\int_{|w| \geq A}\left|f^{*}(w)-g_{\tau, \eta}(w)\right| d w & =\int_{E_{0}^{c}} \int_{A}^{+\infty} g_{\tau, \eta}(\rho s) \rho^{d-1} d \rho d s \\
& \leq c \int_{E_{0}^{c}} \int_{A}^{+\infty} \rho^{-2 \eta(\theta)-1} d \rho d s
\end{aligned}
$$

Then, let us decompose the integral over $E_{0}^{c}$ into the sum of two integrals, one over a set $F_{\delta}=\{s, \eta(s)-H>\delta / 2\}$ defined for $\delta>0$, and the other over a set $E_{\delta}=\{s, 0<\eta(s)-H<$ $\delta / 2\}$. It follows that

$$
\int_{|w| \geq A}\left|f^{*}(w)-g_{\tau, \eta}(w)\right| d w=O\left(A^{-2 H}\right)\left(A^{-\delta}+\mu\left(F_{\delta}\right)\right) .
$$

where $\mu\left(F_{\delta}\right)$ is the measure of $F_{\delta}$ on the unit sphere of $\mathbb{R}^{d}$. But, as shown by Richard (2016b), $\lim _{\delta \rightarrow 0} \mu\left(F_{\delta}\right)=0$. Hence, letting $\delta=\log (A)^{1-\alpha}$ for some $0<\alpha<1$, we obtain

$$
\int_{|w| \geq A}\left|f^{*}(w)-g_{\tau, \eta}(w)\right| d w=o\left(A^{-2 H}\right) .
$$

Consequently, $\Delta_{A}(x)=o\left(A^{-2 H}\right)$ as $A$ tends to $+\infty$.

Proof (Proposition 3) When the increment field is mono-directional, the expression of $\Gamma_{H, v}$ of Equation (16) reduces to

$$
\Gamma_{H, v}(\theta)=\int_{\mathbb{R}^{+}}\left|\hat{v}_{1}(\rho \cos (\theta))\right|^{2} \rho^{-2 H-1} d \rho .
$$

The expression of $\beta_{H, \tau^{*}}$ in Equation (28) follows from the simple coordinate change $u=$ $\rho \cos (\theta)$.

Proof (Theorem 2) We aim at estimating the solution $\tau^{*}$ of a linear system $L_{H} \tau=\beta$ with a random vector

$$
\tilde{\tau}_{\lambda}^{*}=(B+\lambda R)^{-1} L_{H}^{T} \Sigma^{-1} \tilde{\beta}
$$

where $B=L_{H}^{T} \Sigma^{-1} L_{H}, R$ is a diagonal matrix, $\lambda>0$, and $\tilde{\beta}$ is an unbiased estimate of $\beta$ of variance $V(\beta)$. 
Since $\tilde{\beta}$ is unbiased, the expectation of $\tilde{\tau}_{\lambda}^{*}$ satisfies

$$
(B+\lambda R) \mathbb{E}\left(\tilde{\tau}_{\lambda}^{*}\right)=L_{H}^{T} \Sigma^{-1} \beta .
$$

Moreover, $B \tau=L_{H}^{T} \Sigma^{-1} \beta$. Hence,

Thus

$$
(B+\lambda R)\left(\mathbb{E}\left(\tilde{\tau}_{\lambda}^{*}\right)-\tau^{*}\right)=-\lambda R \tau^{*} .
$$

$$
\left|\mathbb{E}\left(\tilde{\tau}_{\lambda}^{*}\right)-\tau^{*}\right|_{2}=\left|(B+\lambda R)^{-1} \lambda R \tau^{*}\right|_{2},
$$

where $|\cdot|_{2}$ denotes the 2 -norm. Hence, using norm properties, we get

$$
\left|\mathbb{E}\left(\tilde{\tau}_{\lambda}^{*}\right)-\tau^{*}\right|_{2} \leq \lambda\left|(B+\lambda R)^{-1}\right|_{2}|R|_{2}\left|\tau^{*}\right|_{2} .
$$

Therefore,

$$
\begin{aligned}
\frac{\left|\mathbb{E}\left(\tilde{\tau}_{\lambda}^{*}\right)-\tau^{*}\right|_{2}}{\left|\tau^{*}\right|_{2}} & \leq \lambda\left|B^{-1}\right|_{2}\left|\left(I+\lambda B^{-1} R\right)^{-1}\right|_{2}|R|_{2} \\
& \leq \frac{\lambda|R|_{2}}{\nu_{-}}\left|\left(I+\lambda B^{-1} R\right)^{-1}\right|_{2},
\end{aligned}
$$

where $\nu_{-}$is the lowest eigenvalue of $B$.

Next, we establish a bound for $\left|\left(I+\lambda B^{-1} R\right)^{-1}\right|_{2}$. For any vector $u$, we have

$$
\left|B^{-1} R u\right|^{2} \geq \frac{1}{\nu_{+}^{2}}|R u|^{2} \geq\left(\frac{1}{\nu_{+}}\right)^{2}|u|^{2} .
$$

Therefore, the lowest eigenvalue of $B^{-1} R$ is above $\nu_{+}^{-1}$. Thus, the one of $I+\lambda B^{-1} R$ is above $1+\lambda \nu_{+}^{-1}$. Consequently,

$$
\left|\left(I+\lambda A^{-1} R\right)^{-1}\right|_{2} \leq \frac{\nu_{+}}{\left(\nu_{+}+\lambda\right)} .
$$

Using Equation (30), we eventually obtain the inequality (24).

Now, we turn to the variance of the estimator. We have

$$
\left.V\left(\tilde{\tau}_{\lambda}^{*}\right)=\mathbb{E}\left(\left(\tilde{\tau}_{\lambda}^{*}-\mathbb{E}\left(\tilde{\tau}_{\lambda}^{*}\right)\right)\left(\tilde{\tau}_{\lambda}^{*}-\mathbb{E}\left(\tilde{\tau}_{\lambda}^{*}\right)\right)^{T}\right)\right)=(B+\lambda R)^{-1} B(B+\lambda R)^{-1} .
$$

Hence,

$$
\operatorname{trace}\left(V\left(\tilde{\tau}_{\lambda}^{*}\right)\right)=\operatorname{trace}\left(\left(I+\lambda B^{-1} R\right)^{-2} B^{-1}\right) .
$$

Moreover, since $B^{-1}$ is a covariance matrix, any term $B_{i j}^{-1}$ of $B^{-1}$ is bounded by $\sqrt{B_{i i}^{-1} B_{j j}^{-1}}$. Hence, it follows that

$$
\operatorname{trace}\left(V\left(\tilde{\tau}_{\lambda}^{*}\right)\right) \leq\left|\left(I+\lambda B^{-1} R\right)^{-1} \Delta\right|_{2}^{2} \leq\left|\left(I+\lambda B^{-1} R\right)^{-1} L_{H}^{T}\right|_{2}^{2}|\Delta|_{2}^{2},
$$

where $\Delta$ is a vector formed by terms $\sqrt{(B)_{i i}^{-1}}$. Noticing that $|\Delta|_{2}^{2}=\operatorname{trace}\left(B^{-1}\right)$ and using Equation (31), we get

$$
\sqrt{\operatorname{trace}\left(V\left(\tilde{\tau}_{\lambda}^{*}\right)\right)}=\frac{\nu_{+}}{\nu_{+}+\lambda} \sqrt{\operatorname{trace}\left(B^{-1}\right)} .
$$

Besides, since $B \tau^{*}=L_{H}^{T} \Sigma^{-1} \beta$, we have

$$
|B|_{2}\left|\tau^{*}\right|_{2} \geq\left|B^{-1 / 2} \Sigma^{-1 / 2} \beta\right|_{2} \geq \sqrt{\nu_{-}} \sqrt{\left\langle\Sigma^{-1} \beta, \beta\right\rangle_{2}} .
$$

Hence,

$$
\frac{1}{\left|\tau^{*}\right|_{2}} \leq \frac{\nu_{+}}{\sqrt{\nu_{-}} \sqrt{\left\langle\Sigma^{-1} \beta, \beta\right\rangle_{2}}} .
$$

Combined with Equation (32), Equation (25) follows.

Proof (Corollary 1) Using expressions of bias and variance in Theorem 2, we clearly see that the function $g$ bounds the relative mean square error. Then, a simple variation analysis of this function suffices to show that it reaches a global minimum at $\lambda^{*}$. 